\title{
Erratum to: Elastic behavior of a red blood cell with the membrane's nonuniform natural state: equilibrium shape, motion transition under shear flow, and elongation during tank-treading motion
}

\author{
Ken-ichi Tsubota $\cdot$ Shigeo Wada $\cdot$ Hao Liu
}

Published online: 29 November 2013

(C) Springer-Verlag Berlin Heidelberg 2013

Erratum to: Biomech Model Mechanobiol

DOI 10.1007/s10237-013-0530-z

In the original publication, the affiliation of the contributors Ken-ichi Tsubota and Hao Liu is published incorrectly. However, the right version is given below.
Page 3, second line from the bottom of the left column: " $\boldsymbol{k B}$ " should not be in bold.

The online version of the original article can be found under doi:10.1007/s10237-013-0530-z.

K. Tsubota $(\varangle) \cdot$ H. Liu

Department of Mechanical Engineering, Chiba University,

1-33 Yayoi, Inage, Chiba 263-8522, Japan

e-mail: tsubota@faculty.chiba-u.jp

K. Tsubota $\cdot$ H. Liu

Shanghai Jiao Tong University and Chiba University International Cooperative Research Center (SJTU-CU ICRC), Chiba University,

1-33 Yayoi, Inage, Chiba 263-8522, Japan

S. Wada

Department of Mechanical Science and Bioengineering,

Osaka University, 1-3 Machikane-yama,

Toyonaka 560-8531, Japan 